\title{
Robust Iris Recognition based on Orientation Field
}

\author{
Pravin S.Patil \\ Department of Electronics and Communication \\ S. S. V. P. S's B. S. Deore College of Engineering, Dhule, (M.S.), India
}

\begin{abstract}
Recently, with an increasing demand on security biometric personal identification has been receiving extensive attention. Among many biometrics techniques, iris recognition is one of the most promising approached due to its high reliability. This paper present a robust iris recognition technique based on orientation field. Initially the eye images have been localized in circular form by using Hough transform. Than flat bed form of iris is generated using Daugman's homogeneous rubber sheet model. From the view point of texture analysis, the local spatial patterns in an iris mainly involve orientation and frequency information. Local image structure plays an important role in iris recognition, so an intuitive idea of iris pattern representation is based on the geometric structure of image data in small region. In our work localized iris image is divided into number of non-overlapping blocks. The blocks orientation has been determined from the pixel gradient orientation based on averaging and optimization. The gradient magnitudes have been computed using Sobel gradient operators. The variance of orientation has been used as feature vector. $\mathrm{L}_{2}$ norm is used to compute a similarity score for the query and template images. The performance of the implemented algorithms has been evaluated using Receiver Operating Characteristics (ROC) and experimental results are reported.
\end{abstract}

\section{General Terms}

Security; Biometrics; Iris Recognition

\section{Keywords}

Iris Image Preprocessing; Orientation field; Feature Variance; Receiver Operating Characteristics

\section{INTRODUCTION}

Security of computer and financial systems plays crucial role nowadays [1][7]. These systems require remembering many passwords that may be forgotten or even stolen. In the second case results are usually disastrous for the user. Thus biometrical based systems are taken into consideration for growing number of applications. Moreover technical progress, along with civilization development, is associated with rising number of dangerous diseases; therefore touch less methods for user authentication, [2] became a very attractive solution. Biometrics recognition[3][4]deals with the uniqueness of an individual arising from their physiological or behavioral characteristics for the purpose of personal identification.(e.g. face, fingerprint, gesture, palm-print, hand geometry, keystroke, rhythms, voice, signature retina, iris) Iris recognition is accepted as one of the most efficient biometric method. The spatial patterns that are apparent in the iris are unique to each individual [5]. Individual differences that exist in the development of anatomical structures in the body result in the uniqueness.[6] The iris is stable, most reliable for personal identification and iris scan is noninvasive.[3][8]Implementing this method to the practical system requires the special image preprocessing where the iris feature extraction plays a crucial role. So far various approaches for iris feature extraction having been proposed by different researchers. [9] [10] [11] [12] [13] [14] [15]. This paper present robust iris recognition algorithms based on orientation field. Section 2, discuss image acquisition. Section 3.desribe image preprocessing which mainly involves Iris localization, eyelashes and eyelids removal, normalization and image enhancement. Section 4 provides introduction to the orientation field for feature extraction. Section 5.discusses iris matching based on feature variance. Experimental results are reported in Section 6, conclusions are drawn in Section 7. In order to implement proposed iris recognition system the basic steps involved have been shown in flow diagram in Figure 1.

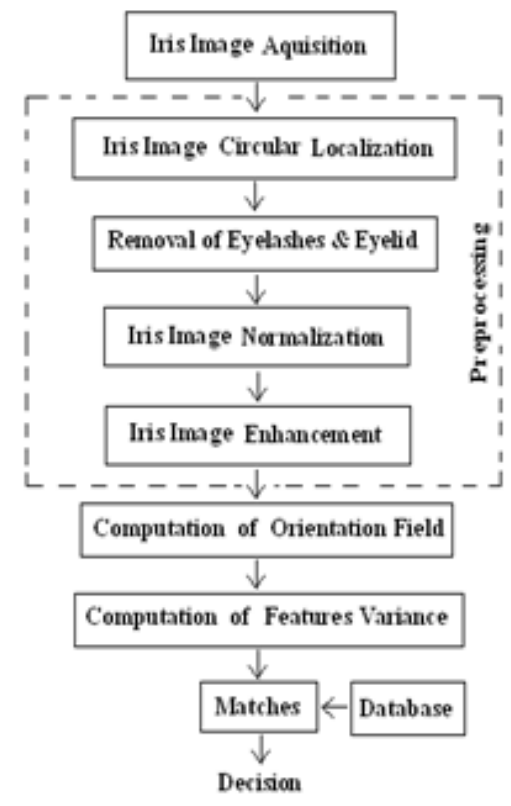

Fig 1: Flow diagram for proposed Iris recognition

\section{IRIS IMAGE ACQUISITIONS}

Capturing iris image of high quality is one of the great challenges [15]. Since iris diameter is fairly small about $1 \mathrm{c} . \mathrm{m}$.Furthermore success of future extraction stage depends upon the iris image quality. When designing an image acquisition apparatus, one should consider three main aspects, the lightning system, the positioning system, and the physical capture system [15] [16] High resolution and good sharpness iris image is acquired by 3CCD camera, which is placed at a distance of approximately 9 c.m. from user eye. The approximate distance between the user and the infrared light is about 12 c.m. which help to reduce specular reflection and capturing rich unique textural information of iris. [18][19]. In our work we use the eye image provided by CASIA (Institute of Automation, Chinese Academy of sciences). These images were taken solely for the purpose of iris software research and implementation [18]. 


\section{IRIS IMAGE PREPROCESSING}

\subsection{Circular Iris localization using Hough transform}

Iris portion can be approximated by two circles, one for the iris/sclera boundary and another, which is interior to the first, forms the iris/pupil boundary. The localization stage is critical to the success of an iris recognition system, since data that is falsely represented as iris pattern data will corrupt the biometric templates generated, resulting in poor recognition rates.[7][15] In this paper we used Hough transform for iris image localization because is the best in account of accuracy. [13]The Hough transform is a standard computer vision algorithm that can be used to determine the parameters of simple geometric objects such as lines and circles present in an image [17]. The circular Hough transform have been employed to deduce the radius and centre coordinates of the pupil and iris regions [20] [21] [22].

Initially, an edge map is generated by calculating the first derivatives of intensity values in an eye image and then thresholding the result. From the edge map, votes are cast in Hough space for the parameters of circles passing through each edge point. These parameters are the centre coordinates $X_{c}$ and $Y_{c}$, and the radius $\mathrm{r}$, which are able to define any circle according to the equation (1) given by,

$X_{c}^{2}+Y_{c}^{2}-r^{2}=0$

A maximum point in the Hough space will correspond to the radius and centre coordinates of the circle best defined by the edge points as, (2)

$$
\begin{aligned}
\left\{-\left(x-h_{j}\right) \sin \theta_{j}+\right. & \left.\left(y-k_{j}\right) \cos \theta_{j}\right\}^{2} a_{j}\left\{\left(x-h_{j}\right) \cos \theta_{j}\right. \\
& \left.+\left(y-k_{j}\right) \sin \theta_{j}\right\}
\end{aligned}
$$

Where, $a_{j}$ controls the curvature, $\left(h_{j}, k_{j}\right)$ are the peak of the parabola, and $\theta_{j}$ is the angle of rotation relative to the $\mathrm{x}$ axis. In performing the preceding edge detection step, we bias the derivatives in the horizontal direction for detecting the eyelids; and in the vertical direction for detecting the outer circular boundary of the iris of the eye images from CASIA $\mathrm{V}_{1}$ iris database. For finding Hough transform, we provide range of iris and pupil radius as range of circle radius from CAISA $V_{1}$ IRIS database specification and create edge map of eye for iris co-ordinates detection and we create edge map of extracted iris region for pupil radius and co-ordinates from Hough transform. Brightest point in Hough transformed image is the desired center for IRIS.

\subsection{Removing Eyelids and Eyelashes}

Removing the portion of the Iris occluded by the eyelids / eyelashes (as can be clearly observed from Figure 2.(f)) is very important because it affects the recognition results.[21] The eyelids / eyelashes are occluding part of the Iris, so only the portion of the image below the upper eyelids and above the lower eyelids are included. This is achieved by changing the gray level above the upper eyelids and below the lower eyelids to ' 0 ' (black). The resultant Iris image after removal of the eyelids / eyelashes has been presented in Figure 3.
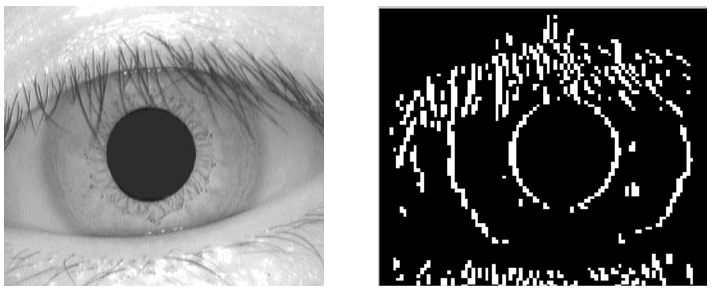

(a)

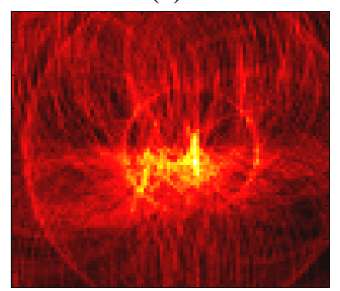

(c)

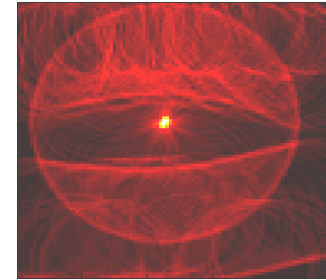

(e) (b)

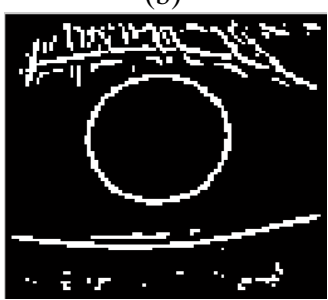

(d)

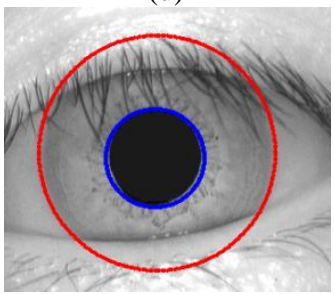

(f)
Fig 2: (a) Original eye image (CASIA: 14_1_1.bmp) from CASIA database,(b) Vertical edge map of eye, (c) Hough transform, (d) Horizontal edge map,(e) Hough transform image showing center of the Iris, (f) Localized Iris image.

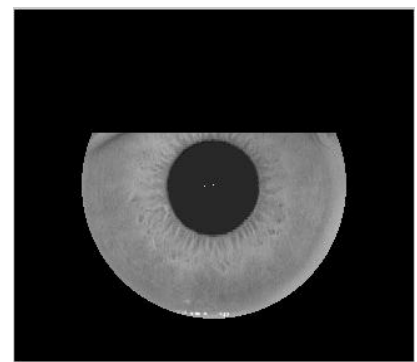

Fig 3: Resultant IRIS after removal of the eyelids / eyelashes.

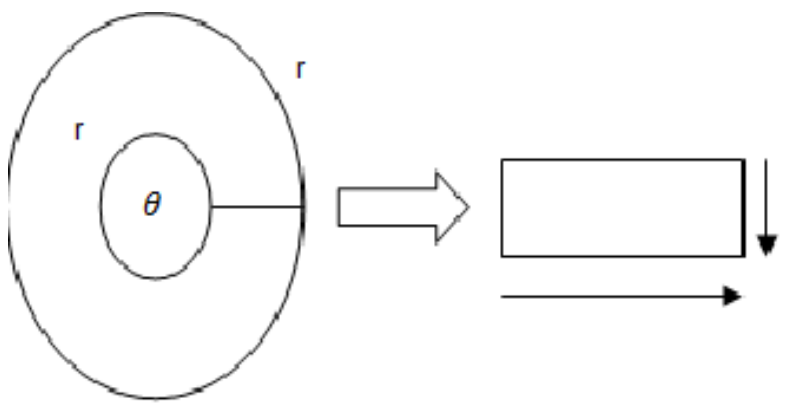

Fig 4: Implementation of Iris unwrapping.

\subsection{Iris Image Normalization}

Flat bed Iris localization is nothing but unwrapping the circular localized Iris into a rectangular region [15]. Image processing of the Iris region is computationally expensive. In addition the area of interest in the image is a 'donut' shape, and grabbing pixels in this region requires repeated rectangular-to-polar conversions. To make things easier, the Iris region is first unwrapped into a rectangular region using simple trigonometry.[23] This allows the Iris decoding algorithm to address pixels in simple (row, column) format. Figure 4 shows simple implementation of the Iris unwrapping. [7]

Once the Iris region is successfully segmented from an eye image, the next stage is to transform the Iris region so that it has fixed dimensions in order to allow comparisons. The 
dimensional inconsistencies between eye images are mainly due to the stretching of the Iris caused by pupil dilation from varying levels of illumination. Other sources of inconsistency include, varying imaging distance, rotation of the camera, non-concentric pupil displacement, head tilt, and rotation of the eye within the eye socket [15].

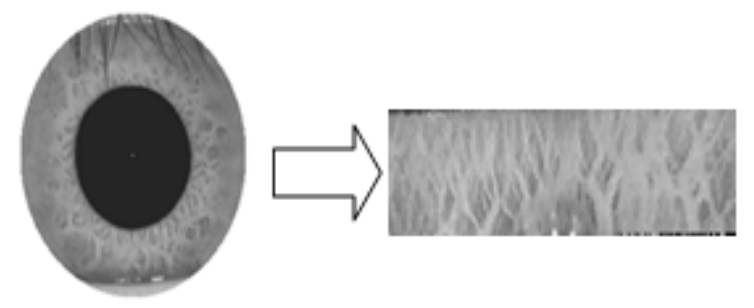

Fig 5: Flat bed Iris localization (a) circular localized IRIS (CASIA V 1 : 33_1_3.bmp) (b) Unwrapped normalize image.

Another point of note is that the pupil region is not always concentric within the Iris region, and is usually slightly nasal. This must be taken into account if trying to normalize the 'donut' shaped Iris region to have constant radius.

The homogenous rubber sheet model can be shown in Figure 4. Relevant to Iris and pupil inside it we can remap each point within the Iris region to a pair of polar coordinates (r, $\theta$ ) where $r$ is on the interval $[0,1]$ and $\theta$ is the angle between $[0$, $2 \pi]$.

The remapping of the Iris region from $(\mathrm{x}, \mathrm{y})$ Cartesian coordinates to the normalized non-concentric polar representation is modeled as,

$$
\begin{gathered}
I(x(r, \theta), y(r, \theta) \rightarrow I(r, \theta) \\
x(r, \theta) \rightarrow(1-r) x_{p}(\theta)+r\left(x_{1}\right) \theta \\
y(r, \theta)=(1-r) y_{p}(\theta)+r y_{1}(\theta)
\end{gathered}
$$

Where, $I(x, y)$ is the Iris region image, $(x, y)$ are the original Cartesian coordinates, $(r, \theta)$ are the corresponding normalized polar coordinates, $x_{p} y_{p}$ are the coordinates of the pupil, and $x_{1}, y_{1}$ are the Iris boundaries along the $\theta$ direction. This procedure of Iris unwrapping is applied on Iris images from the CASIA $V_{1}$ Iris database and the resultant output has been illustrated in Figure 5.

In our experiments for generating the normalized flat bed Iris image, we find that zigzag colleratte area as one of the most important parts of complex Iris pattern, since it is less affected by the eyelids and eyelashes. It is found that colleratte region is generally concentric with the pupil and the radius of this area is restricted in a certain region.[11][24] So we extract the flat bed Iris for the region closer to the pupil as illustrated in Figure 6. This region takes up about $80 \%$ of the normalized image.

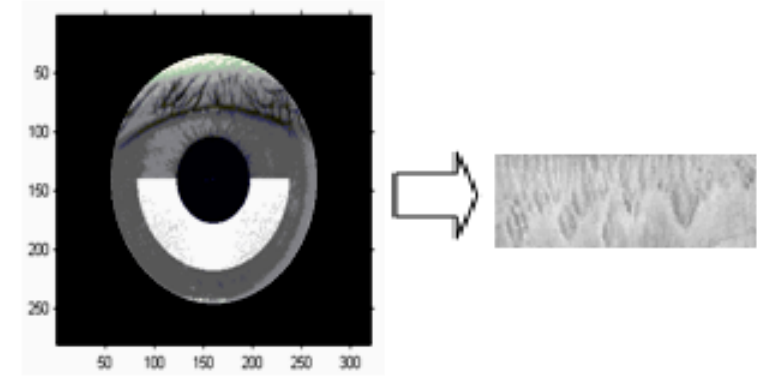

Fig 6: Extraction of flat bed Iris for the region closer to the pupil

\subsection{Iris Image Enhancement}

The unwrapped normalized Iris image still has low contrast and may have non-uniform brightness caused by the position of light sources. All these may affect the subsequent feature extraction and matching. In order to obtain a better distributed texture in the Iris, we first approximate intensity variations across the whole Iris image [13] [15]. The mean of each small block (we have considered the size of each block as $16 \times 16$ empirically) constitutes a coarse estimate of the background illumination. This estimate is further expanded to the same size as that of the normalized image by using bi-cubic interpolation. The resultant estimated background illumination for the same unwrapped normalized Iris image has been illustrated in Figure 7.

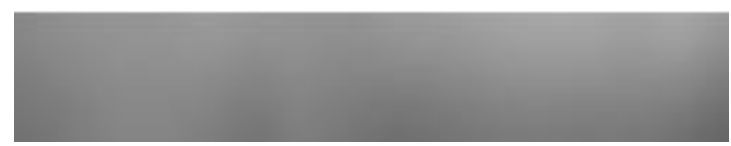

Fig: 7 Estimated background illuminations.

This estimated background illumination is subtracted from the unwrapped normalized image to compensate for a variety of lighting conditions. Then we enhance the lighting corrected image by means of histogram equalization in each $(32 \times 32)$ region. Such processing compensates for non-uniform illumination, as well as improving the contrast of the image. Figure 8 shows the preprocessing result of an Iris image, from which we can see that finer texture characteristics of the Iris become clear.

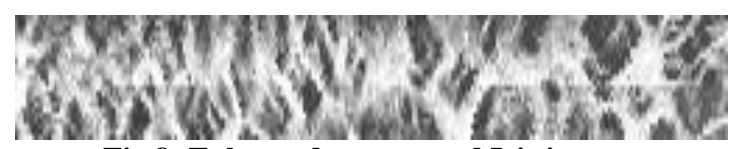

Fig 8: Enhanced unwrapped Iris image.

\section{COMPUTATION OF ORIENTATION FIELD}

The human Iris provided many interlacing minute characteristics such as arching ligaments, ridges, crypts, rings freckles coronas, stripes, zigzag collarets and furrows etc. That can be considered as elementary components of the Iris texture. This visible shape characteristics information is unique to each subject and provides the most discriminating features for Iris recognition.[5][6][8][9][10][11][12][15][16] Thus the pattern of an Iris may be viewed as an oriented texture pattern possessing a fixed orientation and a spatial frequency in a local neighborhood.[13] The fixed orientation is an average orientation and the spatial frequency is due to the inter-shape spacing. It is possible to represent the individuality of Iris based on the local orientation and spatial 
frequency computed for distinct non-overlapping blocks in an Iris image.[12][13][27] To examine the local variations in shape structure, orientations of localized circular or flat bed Iris image have been used as features in the proposed Iris recognition algorithm. Orientations around the pupil in an Iris have been used for image alignment during matching [27] [28]. Smoothing of the localized Iris image is carried out using a low pass filter, which helps in minimizing the effect of noise in gradient computation.

For the computation of orientation field the smoothened localized Iris image is typically divided into number of nonoverlapping blocks and an orientation representative of the shapes in the block is assigned to the block based on analysis of grayscale gradients in the block [25][26]. The block size to be selected depends on the inter-shape distance i.e. it should include at least one shape in a block. The block of size $16 \times$ 16 pixels has been empirically selected for our experimentations. The block orientation could be determined from the pixel gradient orientations based on averaging, voting or optimization. The orientation field of a block $(u, v)$ is given by,

$$
\theta(u, v)=0.5 \tan ^{-1}\left(\frac{V_{x}(u, v)}{V_{y}(u, v)}\right)
$$

Where,

$$
\begin{gathered}
V_{x}(u, v)=\sum_{u=i-\frac{w}{2}}^{i+\frac{w}{2}} \sum_{v=j-\frac{w}{2}}^{j+\frac{w}{2}} 2 G_{x}(u, v) G_{y}(u, v) \\
V_{y}(u, v)=\sum_{u=i-\frac{w}{2}}^{i+\frac{w}{2}} \sum_{v=j-\frac{w}{2}}^{j+\frac{w}{2}}\left(G_{x}^{2}(u, v)-G_{x}^{2}(u, v)\right)
\end{gathered}
$$

Where, $w$ is the size of a block. $G_{x}=\frac{\partial f}{\partial x}$ and $G_{y}=\frac{\partial f}{\partial y}$ are the gradient magnitudes of Iris image $f(x, y)$ in $x$ and $y$ directions respectively. The gradient magnitudes have been computed using Sobel gradient operators. Thus, a $16 \times 16$ size direction matrix of the Iris image is formed as shown in Figure 9

\section{COMPUTATION OF FEATURES}

\section{VARIANCE}

The variance is a measure of energy and this has been used as novel method for deriving the texture features of the Iris images. It could be easily observed that there are substantial discriminating variations in orientation in the Iris image. The variance of orientation has been used as feature vector [29]. The query and template images have been matched using the $\mathrm{L}_{2}$ norm [30].

The variance features are computed as,

$$
\bar{v}=\left[\sigma_{1}^{2}, \sigma_{2}^{2}, \ldots, \sigma_{n}^{2}\right]^{T}
$$

Where,

$$
\sigma_{k}^{2}=\sum_{i=1}^{n}\left[\theta(i, k)-\mu_{k}\right]^{2} \text { for } k=1, \ldots, m .
$$

$\mu_{k}$ is mean of $\mathrm{k}^{\text {th }}$ column of orientation matrix, $m$ and $n$ the number of columns and rows of $\theta(u, v)$ respectively.

Thus a feature vector of size $1 \times 16$ is generated. The variance feature map for two different Iris images are shown in Figure 10. These feature maps are used to represent and match the query Iris image.

\section{EXPERIMENTAL RESULTS AND DISCUSSION}

The algorithm has been implemented and tested on PentiumIV processor with $2.6 \mathrm{GHz}, 512 \mathrm{MB}$ RAM under MATLAB environment. CASIA-Iris $V_{1}$ includes three subsets which are labeled as CASIA-Iris $V_{1}$-Interval, CASIA-Iris $V_{1}$-Lamp, CASIA-Iris $V_{1}$-Twins. From the database we have selected 372 Iris images from 108 different eyes (under different conditions) of 54 subjects. The images in the database have been acquired during different stages and the time interval between two collections is at least one month, which provides a challenge to the algorithm. All Iris images are 8 bit graylevel JPEG files, collected under near infrared illumination.

The performance of the algorithm has been evaluated using the Receiver Operating Characteristics (ROC). This consists of a measure of false acceptance rate (FAR) and the false rejection rate (FRR) at various thresholds. Alternately the Genuine acceptance rate (1-FRR) and FAR may be measured at different thresholds. A genuine matching score is obtained when two feature vectors of the same individual are compared and an imposter matching score is obtained when feature vectors of two different individuals are compared. A single template per subject has been considered for experimentation.

The FAR and FRR for localized circular and flat bed Iris images have been computed using,

False Rejection Rate

$$
\begin{aligned}
& =\left[\frac{\text { True claims Rjected }}{\text { Total true claims }}\right] \\
& \times 100
\end{aligned}
$$

False acceptance rate

$=\left[\frac{\text { Imposter claimed accepted }}{\text { Total imposter claim }}\right]$

$\times 100$

Genuine Acceptance Rate

$$
=1-\% F R R
$$



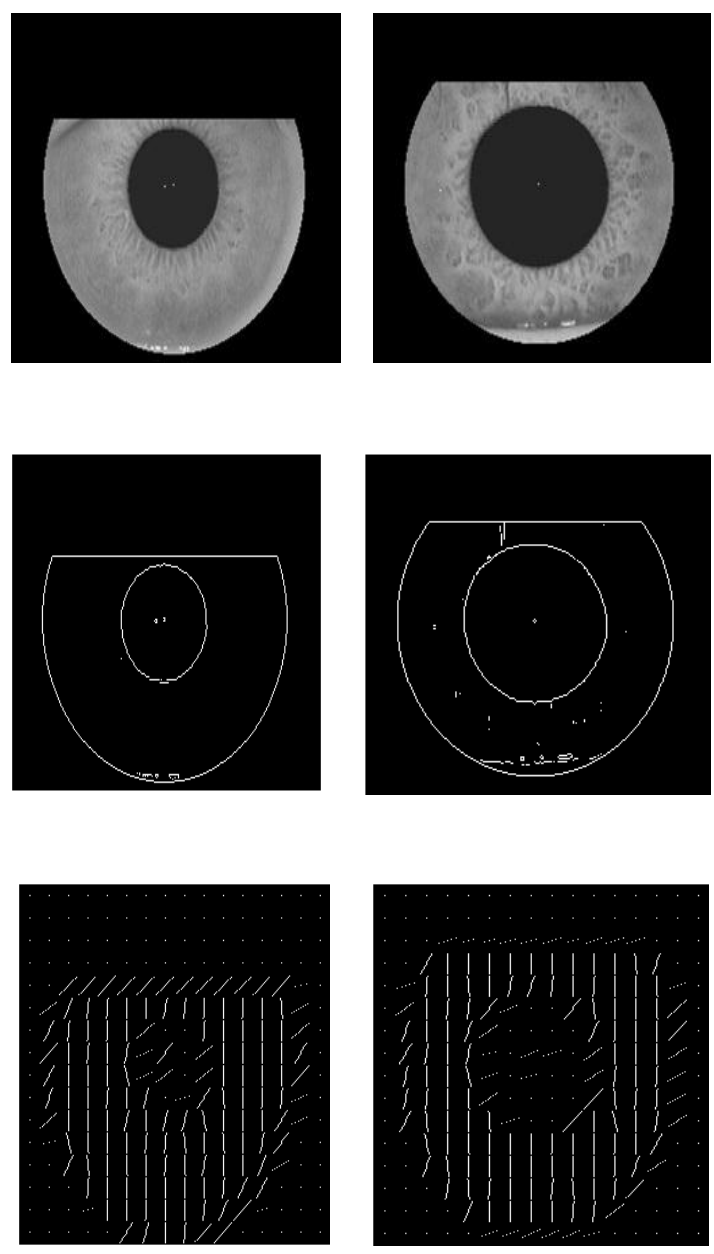

Fig 9: Two different Iris images from CASIA $V_{1}$ Iris database along with their Gradient and orientation images.

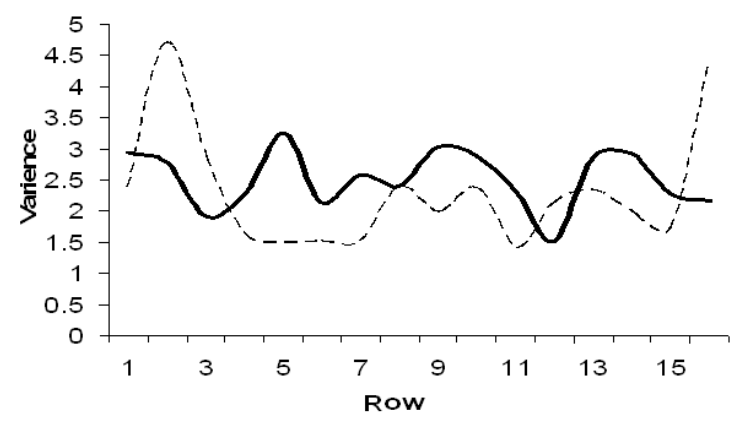

Fig 10: Variance feature histogram of two different Iris images. solid line : Iris 1, dashed line : Iris 2.

The algorithm has been tested for computation of FAR and FRR. FAR and GAR at various values of thresholds for localized circular Iris have been illustrated in Figure 12. The genuine acceptance rate reported $96.33 \%$ at $11.3 \%$ FAR and $78 \%$ for $0.17 \%$ FAR. Results are also reported for various thresholds on localized flat bed Iris in Figure 13.

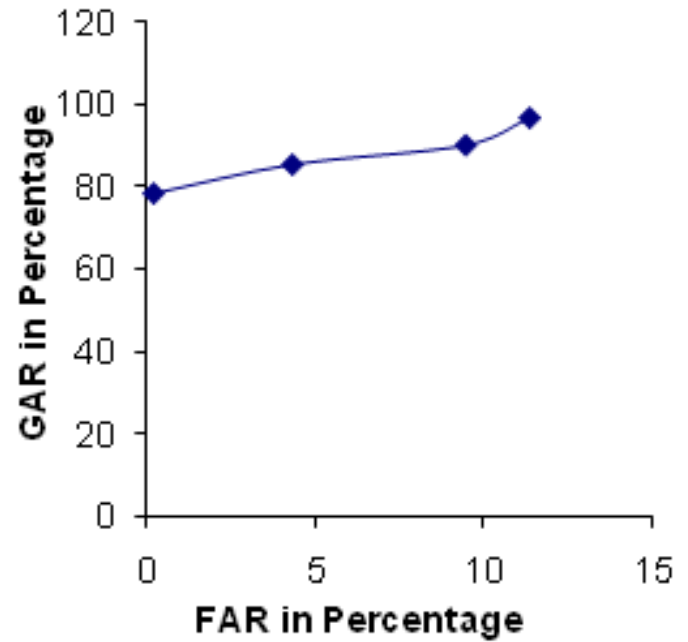

Fig 11: Experimental results for localized circular IRIS

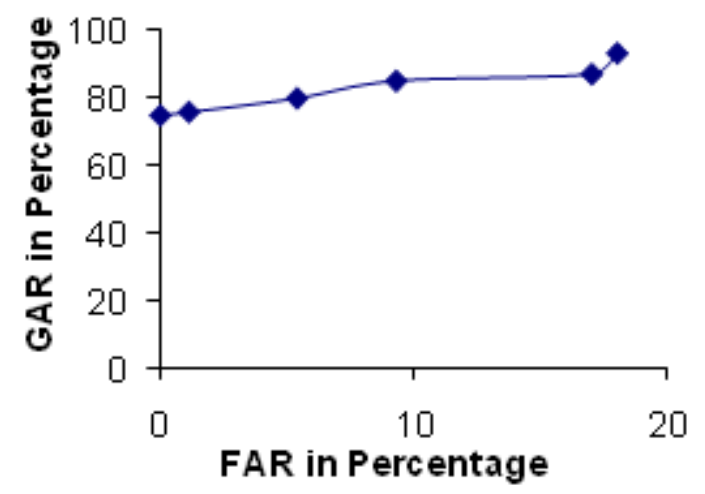

Fig 12: Experimental results for localized flat bed IRIS

\section{CONCLUSION}

This work presented here is simple and effective geometrydriven Iris recognition algorithms. The proposed orientation feature, produce the discrimination between the two Irises of different persons in effective way. The proposed method has ability to estimate the amount of rotation in an Iris image is an efficient way, which takes only few computations. The proposed algorithm produces better results at lower and higher values of FAR.As the algorithm takes fewer computations it could be implemented using the real time processor.

\section{REFERENCES}

[1] A.K.Jain, Ross A.,Prabhakar S."An Introduction to Biometric Recognition",IEEE Transction on Circuits and System for Video Technology-Special issue on Image and Video-Based Biometrics,vol.14,issue.1,2004

[2] S.Sanderson,J.Erbetta,"Authentication for Secure Environments Based on Iris Scanning Technology",IEEE Colloquium on Visual Biometrics,2000

[3] A.K. Jain,R.M.Bolle and S.Pankanti, "Biometric: Personal Identification in a Networked Society", Eds. Norwell MA:Kluswer, 1999

[4] D.Zhang, "Biometrics Technologies and Application",Proceeding of International Conference on Image and Graphics,pp.42-49,Tianjing,China,August 2000 
[5] H.Davision, "The Eye”,Academic,Landon,1962

[6] F.H.Adler,"Physiology of the Eye: Clinical Application,Fourth ed.Landon,The C,V. Mosby Company, 1965

[7] J. Daugman, "How Iris recognition works," Proceeding of International Conference on Image processing, vol. no.1, 2002.

[8] J. Daugman, "High confidence visual recognition of person by a test of statistical independence," IEEE Trans. Pattern analysis and machine intelligence, vol.15,no.11,pp1148-1161, Nov. 1993.

[9] J.Daugman,"Demodulation by Complex-Valued Wavelets for Stochastic Pattern Recognition," Int'l J. Wavelets, Multi-resolution and Information Processing, vol. 1, no. 1 , pp 1-17, 2003.

[10] W.W. Boles, B. Boashah, "A human identification technique using of the Iris and wavelet transform," IEEE Trans. on signal processing vol.46, no.2,pp1185-1188 Apr.1998.

[11] R.P. Wildes, "Iris Recognition: An Emerging Biometric Technology", Proceedings of the IEEE, vol.85, pp.13481363, Sept. 1997.

[12] L Ma, Tieniu Tan, Yunhong Wang," Efficient Iris Recognition by Characterizing Key Local Variations," IEEE Trans. on Image Processing, vol.13, no.6, pp 739750 , June 2004

[13] L. Ma, Y. Wang, T. Tan, "Iris recognition using circular symmetric filters," National laboratory of pattern recognition ,Institute of automation, Chinese academy of sciences, 2002

[14] L. Ma, Y. Wang, and T. Tan, "Iris Recognition Based on Multichannel Gabor Filtering," Proceedings Fifth Asian Conf. Computer Vision, vol. I, pp 279-283, 2002.

[15] L Ma, Tieniu Tan, Yunhong Wang," Personal Identification Based on Iris Texture Analysis," IEEE Transaction on Pattern analysis and machine intelligence, vol.25, no.12, pp 1519-1533, December 2003.

[16] R. Wildes, J. Asmuth, G. Green, S. Hsu, R. Kolczynski, J. Matey, S. McBride, "A Machine-Vision system for automated Iris recognition," Proceeding Machine Vision and Application,vol.9,pp 1-8,1996

[17] L. Masek, "Recognitions of Human Iris Patterns for Biometric Identifications [B]", School of Computer Science and Software Engineering, The University of Western Australia, 2003.
[18] "CASIA Iris Image Database", Institute of Chinese Academy of Sciences, http://www.sinobiometrics.com/2004

[19] M. Ezhilarasan, R.Jacthish, K.S. Ganbathy Subramaniam, R.Umapathy "Iris Recognition Based on its Texture Patterns" International Journal on Computer Science and Engineering,vol.2,issue.9,pp.30713074,2010

[20] R. Wildes, J. Asmuth, G. Green, S. Hsu, R. Kolczynski, J. Matey, S. McBride, "A system for automated Iris recognition," Proceeding IEEE Workshop on Application of Computer Vision,Sarasota,FL,pp.121-128,1994

[21] W. Kong, D. Zhang, "Accurate Iris segmentation based on novel reflection and eyelashes detection model," Proceeding of 2001 International symposium on intelligent multimedia, video and speech processing Hong Kong, 2001.

[22] C. Tisse, L. Martin, L. Jorres, M. Robert, "Person identification technique using human Iris recognition," International conference on vision interface Canada, 2002.

[23] Iris Recognition:Unwrapping the Iris http://cnx.org/content/m12492/latest, 26/10/2006

[24] H.Sung, J. Lim, Y. Lee, "Iris recognition using collarette boundary localization," Proceeding of the $17^{\text {th }}$ International conference on pattern recognition (ICPR04)

[25] P.Perona, “ Orientation Diffusion”,IEEE Transction on Image Processing,vol.7,pp.457-467,1998

[26] P.E.Trahanias and A.N.Venetsanopoueos " Vector Directional Filters", IEEE Transction on Image Processing,vol.2,pp 528-534,1993

[27] S.Noh,K.Boe and J.Kim, " A Novel Method to Extraction Features for Iris Recognition System" AVBPA2003,pp.838-844

[28] Z.Sun,Y.Wang,T.Tan,J.Cui “ Robust Direction Estimation of Gradient Vector Field for Iris Recognition" IEEE International Conference, 2004

[29] G.Hong-Ying,Z.Yue-ting,P.Yun-he “An Iris Recognition Method Based on Multi-Orientation Features and NonSymmetrical SVM, Journal of Zhejiang University SCI 2005 6A(5):428-432

[30] C.Sanchez-Avila and R.Sanchez-Reillo, "Two Different Approaches for Iris Recognition using Gabor Filters and Multiscale Zero-crossing Representation", Journal of the Pattern Recognition 38,pp.231-240, 2005 